\title{
Mucinous cystadenoma of the retroperitoneum, laparoscopy or an open approach? Two case reports and review of the literature
}

\author{
Ory Wiesel • David Spector • Joseph M. Klausner • \\ Boaz Sagie
}

Received: 15 January 2009 / Accepted: 17 February 2009/Published online: 7 March 2009

(C) Springer-Verlag 2009

\begin{abstract}
Big cystic lesions of the retroperitoneum are rare entities. Most of the cases involved females of reproductive age and were related to ovarian structures. The recommended treatment is surgery, and more than $90 \%$ of the reported cases were resected in an open midline abdominal incision. We present two patients who were referred to our clinic due to abdominal mass that was causing vague abdominal pain. Homogenic retroperitoneal cystic mass was demonstrated on computerized tomography (CT). One was resected by laparoscopy and the other by laparotomy, both revealed a mucinous cystadenoma of the retroperitoneum. A review of the literature is also presented.
\end{abstract}

Keywords Cystic lesion of retroperitoneum .

Mucinous cystadenoma

\section{Introduction}

Retroperitoneal mucinous cystadenoma is a very rare entity, with several dozen having been reported in the English literature since it was first described in 1989 [1]. Most of the cases involved females of reproductive age and were related to ovarian structures. The recommended treatment is surgery, and more than $90 \%$ of the reported cases were

\footnotetext{
O. Wiesel · D. Spector · J. M. Klausner · B. Sagie Division of Surgery "B", Tel Aviv Sourasky Medical Center, Sackler Faculty of Medicine, Tel Aviv University, Tel Aviv, Israel

O. Wiesel $(\bowtie)$

Department of Surgery "B", Tel Aviv Sourasky Medical Center, Tel Aviv University,

6 Weizman Street,

Tel Aviv 64239, Israel

e-mail: seker13@gmail.com
}

resected in an open midline abdominal incision. We present two patients who were referred to our clinic due to abdominal mass that was causing vague abdominal pain. Homogenic retroperitoneal cystic mass was demonstrated on computerized tomography (CT). One was resected by laparoscopy and the other by laparotomy, both revealed a mucinous cystadenoma of the retroperitoneum.

\section{Case 1}

A 43-year-old woman was referred to our clinic with vague abdominal pain and a palpable mass on the left lower quadrant (LLQ) of her abdomen. Her past medical history was significant for a left kidney cortical cyst that had been detected 3 years earlier, a fibroma in her right femoral region, and bilateral axillary lipomas, all of which had been surgically removed. Upon physical examination, her abdomen was soft, without peritoneal signs, and a $10-\mathrm{cm}$ tender mass was palpated on her LLQ and middle abdomen. A digital rectal examination was normal, as was a gynecologic examination. CT of the abdomen revealed a homogenic retroperitoneal cystic mass measuring $16 \times 7.8 \times 13 \mathrm{~cm}$ on her LLQ, with subtle borders in the proximity of the left ureter and sigmoid colon and enlarged retroperitoneal lymph nodes. The mass could be seen pushing the left colon. Bilateral ovarian cysts were also demonstrated on CT (Fig. 1). Her blood count, electrolytes, liver, kidney functions, CEA, and CA19-9, CA125 levels were all within normal limits.

The patient was taken to diagnostic laparoscopy during which on-table decision of whether to continue to laparotomy or not was planned. A left-sided ureter catheter was inserted to avoid any missed injuries to the ureter prior to the laparoscopic exploratory procedure. The laparoscopic 


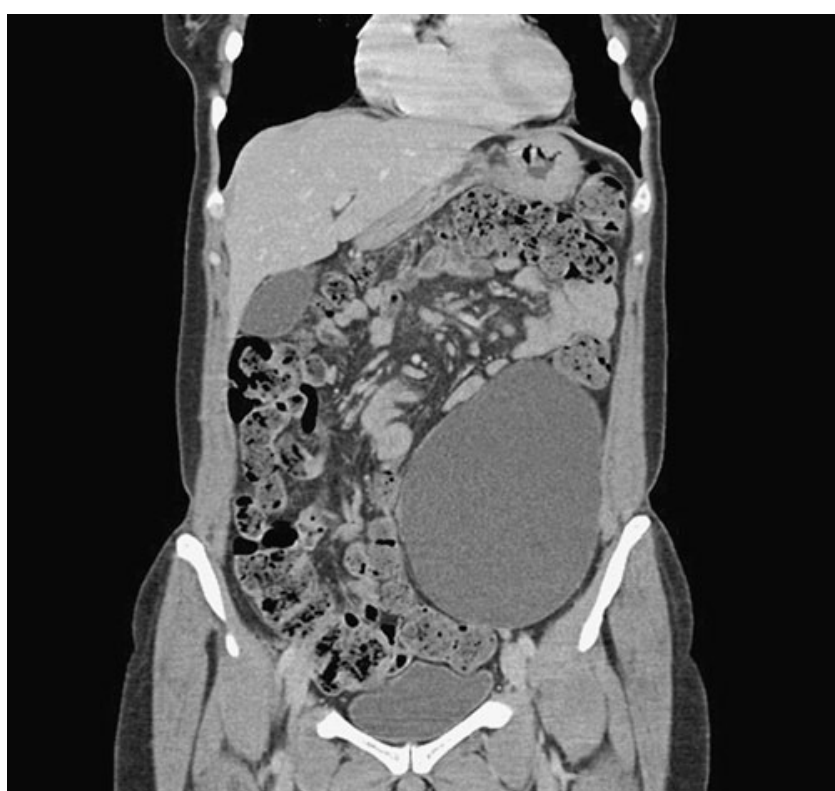

Fig. 1 First patient's CT scan of the cyst prior to the surgery. Note that the cyst is separated from nearby organs but seemed attached to the left ovary

exploration was initiated by insertion of a Veress needle in the right subcostal region in order to avoid injury to the cyst. After insufflation of the peritoneal cavity with $\mathrm{CO}_{2}$, an abdominal exploration revealed a normal-looking liver, bowel, a small cyst on the right ovary, and the retroperitoneal huge cyst which was near the anterior side of the sigmoid colon in the LLQ, and clearly unattached to the parenchymatic organs around it (i.e., the colon, left kidney and ureter, and ovaries).

Isolation of the cyst from the rest of the organs was done, followed by aspiration of the cyst fluid which was noted as being clear and serous. No frozen sections were

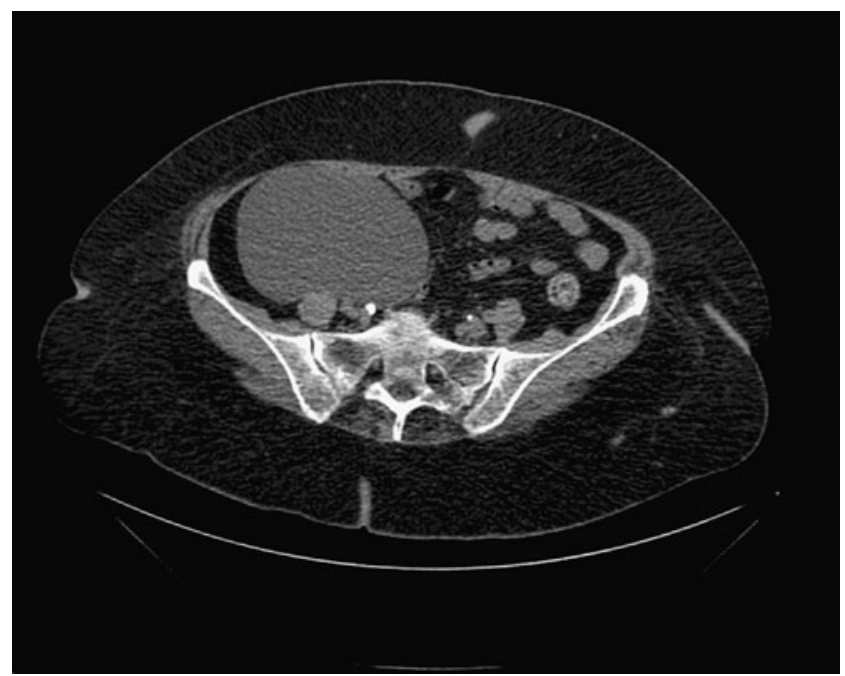

Fig. 2 Second patient's CT scan prior to surgery sent for surgical staging. The cyst was removed with a nylon bag via the left upper trocar port site.

The postoperative course was uneventful and the patient was discharged home on postoperative day 2 .

The pathologic report described a retroperitoneal mucinous cystadenoma with borderline areas. A serous lining was also present. The specimen was positive for CK7, partially positive for CEA, CA125, CK20 calretinin, negative for CK5/6, and positive for Ki67 in 10\% of the basal layer cells. Histochemical stains for PAS, PAS-diastase and Alcian blue were all positive.

\section{Case 2}

A 76-year-old female was referred to our clinic duo to vague abdominal pain 4 years after transvaginal hysterectomy duo to benign lesion. Her physical examination revealed soft abdomen with right lower abdominal mass. Her blood count, electrolytes, liver, kidney functions, tumor markers were all within normal limits. Abdominal ultrasound found cystic lesion in the right abdomen. Contrast-enhanced computed tomography $(\mathrm{CT})$ revealed $10 \times 8 \times 11-\mathrm{cm}$ cyst in the right ileac fossa with subtle borders with clear fluid. No suspicious lymph nodes were detected. We suspect an ovarian malignancy hence laparotomy was chosen.

Upon laparotomy, a well-defined cystic lesion was found in the retroperitoneum, adjacent to the cecum, mesentery, and the aorta. The mass was separated from the right ovary. After meticulous separation of the cyst from adjacent

Table 1 Classification of lesions of the retroperitoneum as neoplastic and non- neoplastic

\begin{tabular}{ll}
\hline Neoplastic & Cystic lymphangioma \\
Cystic teratoma & Cystic mesothelioma \\
Epidermoid cyst & Mucinous cystadenoma \\
& Mucinous cystadenocarcinoma \\
Tailgut cyst & Perianal mucinous carcinoma \\
& Pseudomyxoma retroperitonei \\
& Urogenital cysts (pronephric, mesonephric, \\
& metanephric, Mullerian cyst) \\
& Cystic lesion in solid tumors (paraganglioma, \\
& neurogenic tumor, gastrointestinal stromal \\
tumor, soft tissue sarcoma, etc.) & Pseudocyst (pancreatic, non-pancreatic) \\
Urinoma & Hematoma \\
Lymphocele
\end{tabular}


organs, an en-block resection of the mass was done without disruption of its capsule. Postoperative period was uneventful. On pathology, benign mucinous cystadenoma lined with fibrocollagenous wall was found, the inner lining of the cyst was formed by single, non-stratified layer of tall columnar mucous secreting cells, cellular stratification, and cellular pleomorphism are absent, no evidence of malignancy was found (Fig. 2).

\section{Discussion}

Cystic lesions of retroperitoneum are rare clinical entities, among which primary mucinous cystadenoma is even more rare [2]. These lesions can be classified as neoplastic and non-neoplastic (Table 1). With the exception of cystic lymphangiomas, which are more frequent in men, most cystic retroperitoneal lesions are found in women aged 1786 years (median 42.3 years) [3]. The patients tend to present with vague abdominal pain that sometimes radiates to the back or leg and may be mistaken for a classical orthopedic problem until an abdominal CT scan verifies the true origin.

A contrast-enhanced abdominal CT scan is ideal for differentiating among the subtypes of the retroperitoneal cysts, although there is substantial overlap among some of them. The correct diagnosis can be arrived at by adding the clinical presentation, gender and age of the patient to the CT scan results. Aspiration of the cyst's fluid may help to define the nature of the fluid, but not the type of epithelial lining of the cyst. Furthermore, it may disseminate neoplastic cells along the needle track and put the patient at risk of metastatic spread.

Surgery is the preferred treatment for most cystic retroperitoneal masses [4].

Since it is not always possible to histologically determine the nature and type of the cyst preoperatively, exploratory laparotomy or, in specific cases, diagnostic laparoscopy is recommended. This is the third reported laparoscopic excision of mucinous cystadenoma. Laparoscopy affords less morbidity and a shorter hospital stay, and may be well suited for excising retroperitoneal cysts, while laparotomy is preferred in cases of suspected malignancy. Spillage of the cystic fluid should be avoided during both procedures.

Primary mucinous cystadenomas are extremely rare and their histogenesis is not understood completely $[5,6]$. This pathology tends to be more frequent in females, and it is commonly accepted that invagination of the peritoneal mesothelium undergoes mucinous metaplasia and further cystic formation. Malignant transformation of primary mucinous cystadenomas into cystadenocarcinoma has also been considered several other hypotheses include the suggestion that the tumor arises from ectopic ovarian tissue (which may explain the female tendency), from remnants of embryonic urogenital apparatus, and from a teratoma in which the mucinous epithelium overrides the other teratomatous components and dominates into cystic lesion [7, 8]. Although most of the reported cases were benign, close follow-up is recommended for borderline lesions due to their malignant propensity.

Interestingly, our first patient had both a femoral fibroma and bilateral axillary lipomas which were excised several years before the current presentation. Our literature search yielded no prior report on a link between these three pathologic entities.

In conclusion, this case indicates a need for heightened awareness of primary mucinous cystadenomas among both practicing surgeons and gynecologists and GPs to whom female patients are very likely to consult for the initial symptoms of lower abdominal pain. CT scans are highly informative for differential diagnosis although not always can differentiate the cysts origin and predict its malignancy potential, while laparoscopy can effectively and safely remove the lesion when there is no suspicion of malignancy.

\section{References}

1. Pennell TC, Gusdon JP (1989) Retroperitoneal mucinous cystadenoma. Am J Obstet Gynecol 160:1229-1231

2. Yang DM, Hwang HY et al (2004) Retroperitoneal cystic masses: $\mathrm{CT}$, clinical, and pathologic findings and literature review. Radiographics 24:1353-1365

3. Bifulco G, Mignogna C et al (2008) Huge primary retroperitoneal mucinous cystadenoma of borderline malignancy mimicking an ovarian mass: case report and review. Anticancer Res 28 (4C):2309-2315

4. Chen JS, Chang YJ et al (1998) Laparoscopic resection of a primary retroperitoneal mucinous cystadenoma: report of a case. Surg Today 28:343-345

5. Cottrill HM, Roberts WS (2007) Primary retroperitoneal mucinous borderline tumor: A case report. Gynecologic oncology 106:626627

6. Matsubara M, Kawaguchi K et al (2005) Primary retroperitoneal mucinous cystadenoma of borderline malignancy: a case report and review of the literature. Int J Gynecol Pathol 24:218-223

7. Pearl ML, Chumas J et al (1996) Primary retroperitoneal mucinous cysadenocarcinoma of low malignant potential a case report and literature review. Gynecol Oncol 61:150-152

8. Lai ECH, Lau WY et al (2006) Primary retroperitoneal mucinous cystadenoma. ANZ J Surg 76:547 